\title{
Prevention of complications after inferior myocardial infarction treated by using percutaneous coronary intervention - case report
}

\author{
Petru Bălăceanu1, Nicoleta Dima*,2, Mariana Floria ${ }^{1,2}$, Madalina Ionela Chiriac ${ }^{1}$, \\ Alexandru Mărian' ${ }^{1}$, Ciprian Rezuş ${ }^{1,2}$ \\ 1 "Grigore T. Popa" University of Medicine and Pharmacy, lasi, Romania, ${ }^{2} 3^{\text {rd }}$ Department of Internal \\ Medicine, "Sf. Spiridon" Clinical Emergency Hospital, Iasi, Romania
}

\begin{abstract}
We present the case of a 59 year-old female diagnosed with hypertension and dyslipidemia, who had previously suffered an inferior myocardial infarction, treated by using percutaneous coronary intervention (PCl). The patient subsequently developed hypertensive encephalopathy phenomena, due to an episode of respiratory infection. The hypertensive encephalopathy is a complex clinical entity that requires quick acknowledgment and proper management in order to decrease the high mortality rate. The diagnostic delay would lead to a negative prognosis for the patient, through the development of brain lesions, often irreversible.
\end{abstract}

Keywords: hypertension, hypertensive encephalopathy, myocardial infarction, atherosclerosis

\section{Introduction}

Hypertension represents the fundamental variable risk factor of all cardiovascular pathology [1]. In the Framingham study, the relative risk for developing a stroke is 3.1 in males and 2.9 for females in subjects with blood pressure values higher than 160/95 $\mathrm{mmHg}$. The severity of hypertension is indicated by complications of target organs: brain, heart and kidney [2, 3]. Hypertension can also lead to multiple neurological complications such as: hypertensive encephalopathy, stroke, vascular dementia. The correct administration of antihypertensive drugs is associated with reduced risk of myocardial infarction, stroke, heart failure and cardiovascular death.

Received: August 2014; Accepted after review: September 2014; Published: September 2014

${ }^{*}$ Corresponding author: Nicoleta Dima, $3^{\text {rd }}$ Department of Internal Medicine, "Sf. Spiridon" Clinical Emergency Hospital, lasi, Romania. Email: nicoleta2006r@yahoo.com
Ischemic heart disease, including acute myocardial infarction (AMI), is the most common and severe consequence of hypertension [4, 5]. The atherosclerosis is a progressive disease, which can be influenced in various stages, confirmed by clinical studies (e.g. REVERSAL - Reversal of Atherosclerosis With Aggressive Lipid Lowering, and ASTEROID Study to Evaluate the Effect of Rosuvastatin on Intravascular UltrasoundDerived Coronary atheroma Burden) $[6,7]$.

\section{Case presentation}

We present the case of a 59 year-old female from countryside, retired, with cardiovascular risk factors (hypertension and dyslipidemia), who was admitted to the emergency department for muco-purulent expectoration, cough, exertional dyspnea, headache, palpitations and asthenia. 
These symptoms have suddenly appeared three days prior to hospital presentation and the patient attempted self-medication without improving the overall condition. As for the past medical history, we mention that the patient had suffered an inferior myocardial infarction with right ventricular lesions (July 2013), treated using $\mathrm{PCl}$ with positive clinical outcome following the procedure.

The ischemic heart condition associated with incorrect administration of antihypertensive drugs (the maximal blood pressure - BP around $200 \mathrm{mmHg}$ ) lead to the development of hypertensive encephalopathy phenomena, requiring hospitalization. Physical examination at admission revealed a body mass index of $29.2 \mathrm{~kg} / \mathrm{m}^{2}$, a moderately influenced general condition, without fever, mucocutaneous pallor, sweaty skin, pharyngeal hyperemia, cough and dyspnea with polypnea.
Other pathological findings include a peripheral capillary oxygen saturation $(\mathrm{SpO} 2)=$ $93 \%$, disseminated bronchial rales on both anterior and posterior thorax lung areas, $\mathrm{BP}=$ $180 / 90 \mathrm{mmHg}$, heart rate $(H R)=82$ beats $/ \mathrm{min}$, regular heart rhythm, murmur of aortic calcification, $2^{\text {nd }} / 4^{\text {th }}$ grade of mitral holosystolic murmur, distended abdomen due to adipose tissue, meteorism, flatulence, and liver edge palpable at the costal margin.

It is important to mention that the values of home self-monitored blood pressure were increased $(B P=185 / 90 \mathrm{mmHg})$.

An ECG examination was performed at admission (Figure 1) with the following findings: sinus rhythm, intermediate QRS axis, HR = 79/minute, QS in DIII, inverted T wave in DIII, aVF with izoelectric $T$ in $\mathrm{qR}$ aVF and inverted $T$ wave in $\mathrm{V} 1$.

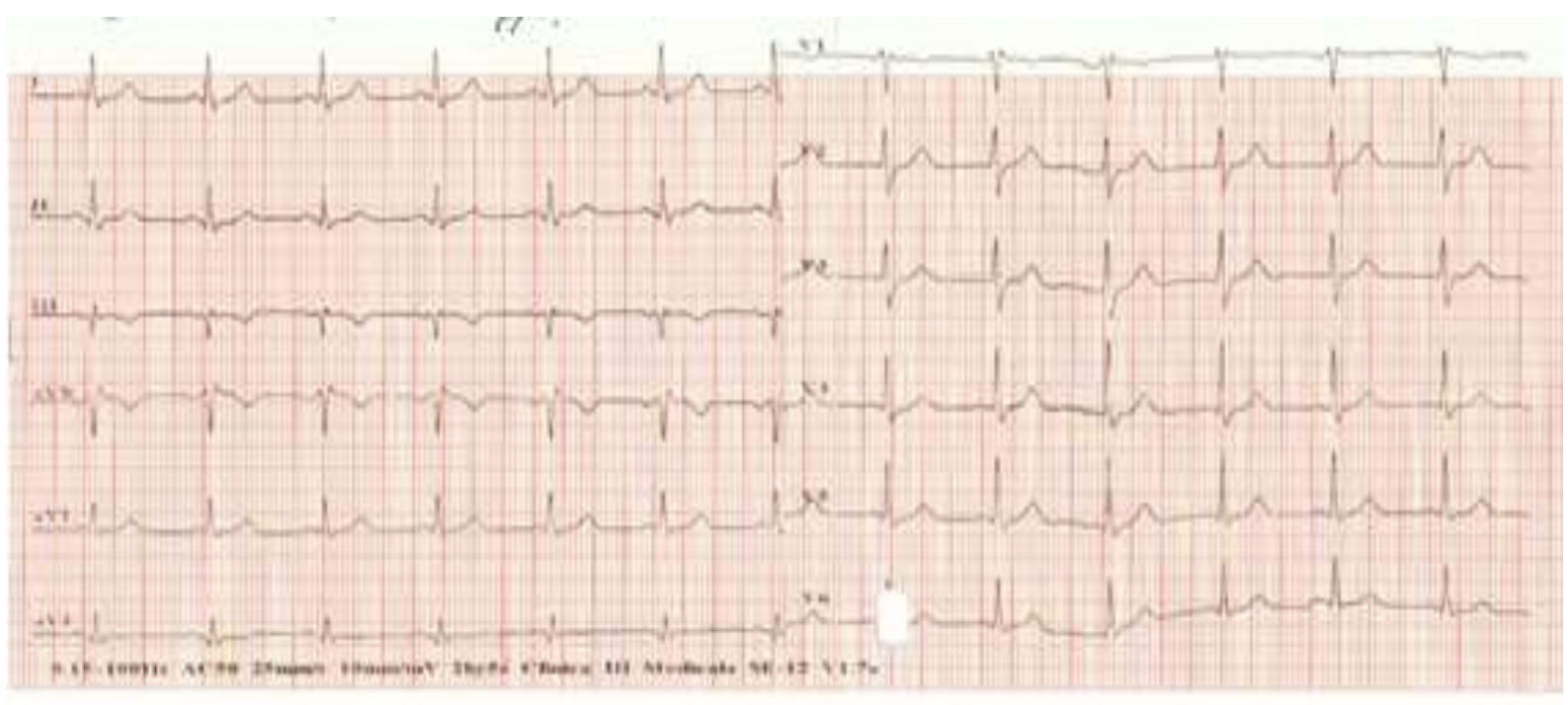

Fig. 1. The Electrocardiography of the patient at admission

The biological investigations revealed neutrophil leucocytosis, normochromic normocytic anemia, postprandial hyperglycemia and important inflammatory syndrome. The serology for hepatitis $B$ virus and hepatitis $C$ virus were negative (Table 1); urine culture was also negative. The chest $\mathrm{X}$-ray examination revealed a cardio-thoracic index of 0.48 , with calcification of aortic knob and normal lung aspect (Figure 2).
The echocardiography findings include normal heart dimension, hypokinetic posterior wall with delayed relaxation, diastolic dysfunction, interventricular septum $=11 \mathrm{~mm}$, left ventricular posterior wall $=11 \mathrm{~mm}$, ejection fraction $=55 \%$ and fractional shortening $=28 \%$. The abdominal ultrasound examination described liver steatosis, hyperechoic aspect of the pancreas without other changes. 
Table 1. The biological investigation results

\begin{tabular}{lcc}
\hline & Biological investigations \\
\hline & & \\
\hline White blood cells/mmc & Admission & Discharge \\
\hline Neutrophils (\%) & 11200 & 7430 \\
\hline Hemoglobin (g/dl) & 74,6 & 46,3 \\
MCV (fL) & 11,8 & 11,3 \\
MCHC (g/dl) & 92,8 & 92,7 \\
Erythrocyte sedimentation rate & 33,7 & 33,1 \\
(mm/h-2h) & $54 / 96$ & $64 / 98$ \\
Fibrinogen (mg/dl) & 517 & 330 \\
C reactive protein (mg/dl) & 1,00 & 0,40 \\
\hline Blood glucose (mg/dl) & 146 & 104 \\
\hline Hepatitis B surface Antigen & Negative \\
\hline Hepatitis C Antibodies & Negative & \\
\hline
\end{tabular}

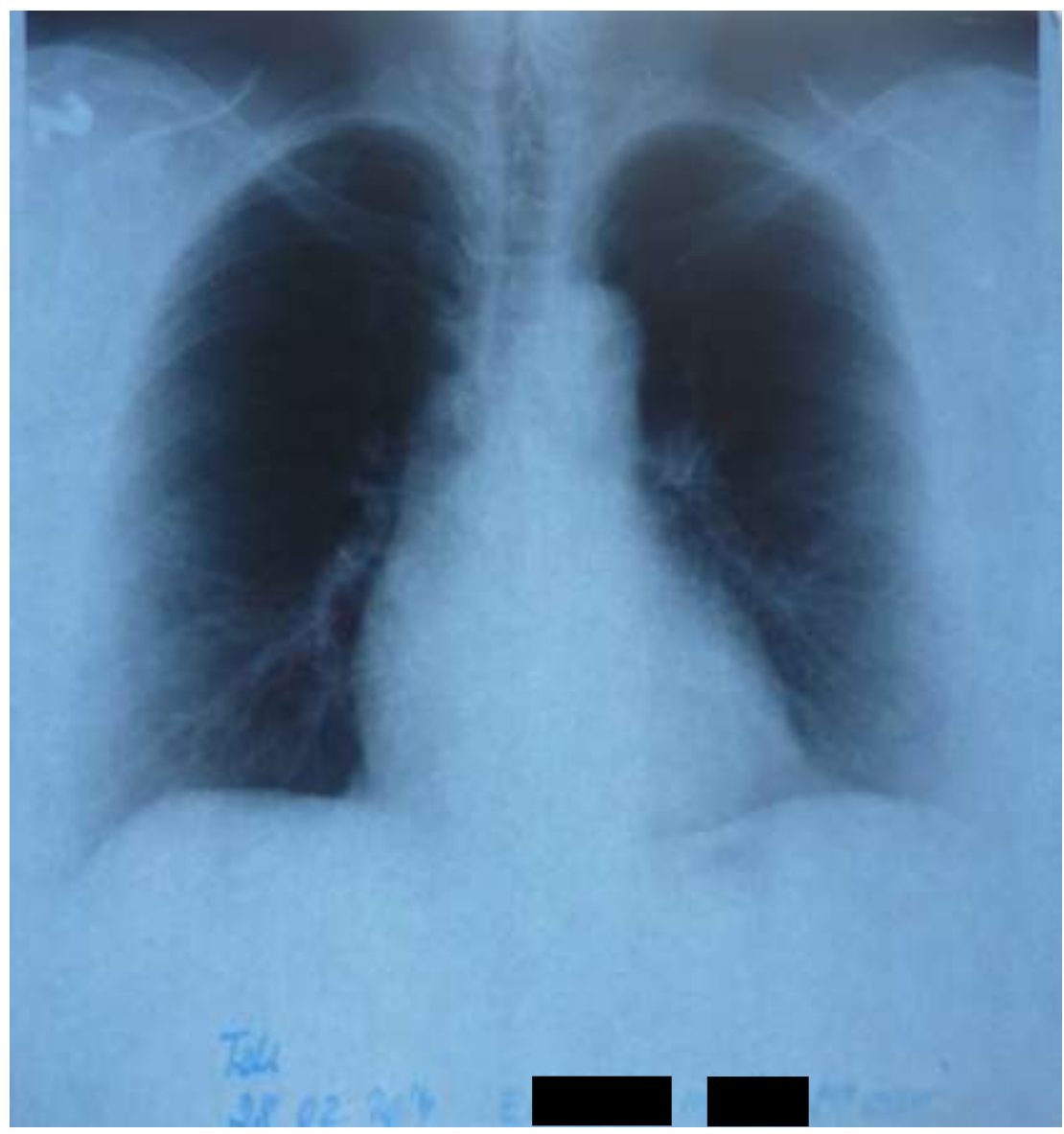

Fig. 2. The aspect of chest $\mathrm{X}$-ray examination

Consequently, antimicrobial therapy with Clarithromycin (500 mgx2/day), coronary vasodilator drugs, symptomatic treatment and reevaluation/modulation of the background medication therapy were established.
Subsequently, the patient showed significant improvement of the clinical outcome and increased exercise tolerance after the 24 hours of hospitalization, with favorable evolution of $\mathrm{BP}$ at review (Figure 3). 


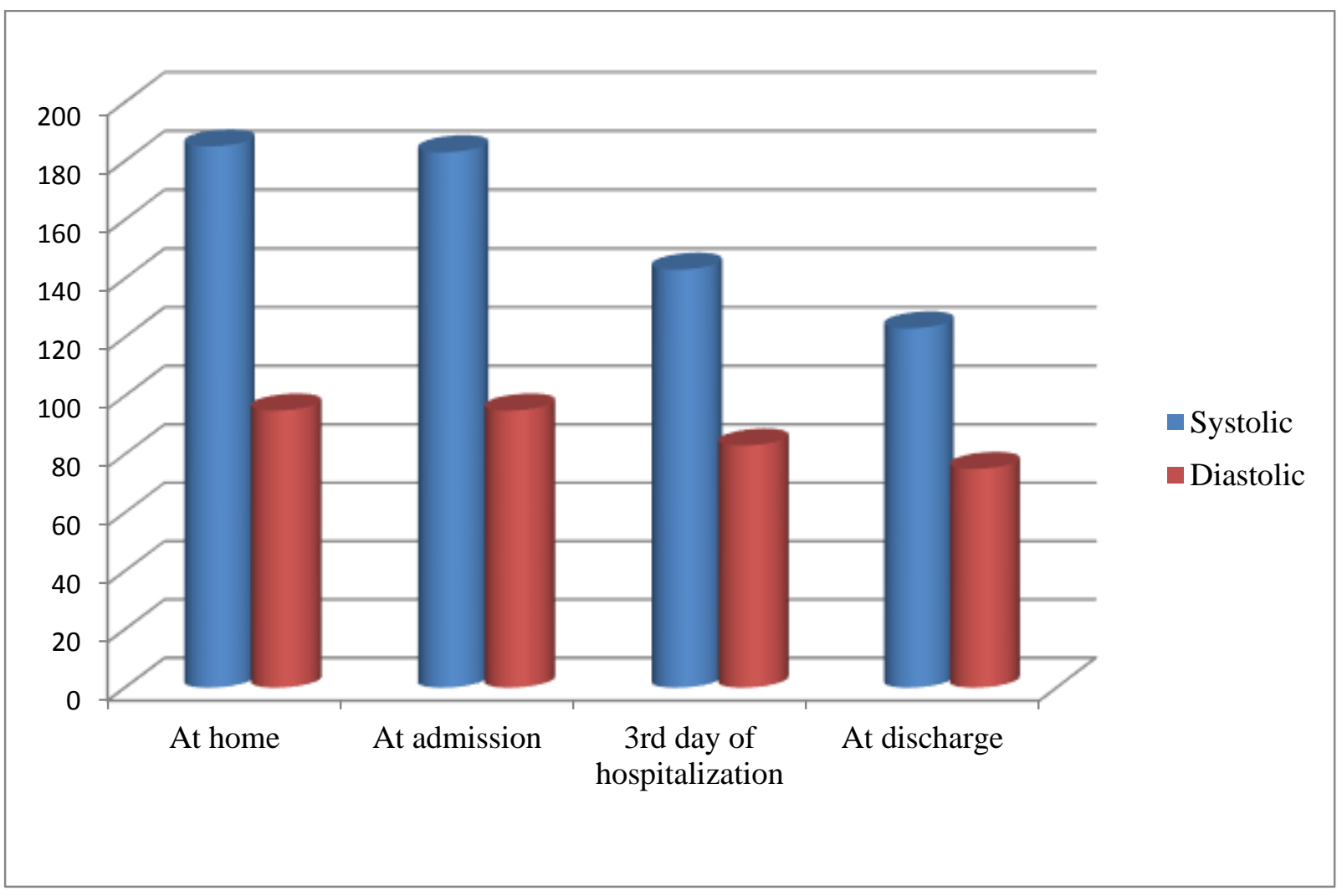

Fig. 3. The evolution of BP

\section{Discussion}

The association of myocardial ischemia causing an acute coronary episode (which needs prompt appropriate treatment) with a hypertensive pattern requires a comprehensive approach of the patient with presumed vasculopathy (due to atherosclerotic processes of coronary and other systemic arteries).

Thus, it is essential to initiate a plurivalent drug therapy in order to stabilize the atherosclerotic plaques and achieve rheological improvement (antiplatelet complex), as well as to include: vascular protective drugs (vasodilators drugs have an important role in vascular remodeling), medication lowering tissue oxygen consumption and antiarrhythmic drugs (beta-blockers in the presented case) [8].

The involvement of acute lung diseases in patients with cardiovascular disease may be correlated with the presence of systemic inflammatory syndrome that may aggravate the coronary atherosclerosis.

\section{Conclusions}

The therapeutic decision has to be based on both clinical and biological evidences, taking into account the possible drug interactions and contraindications.

Our patient was diagnosed with very high additional risk hypertension, thus requiring maintaining the BP values between 130 to 139 $\mathrm{mmHg}$ for systolic BP and between 80 to 85 $\mathrm{mmHg}$ for diastolic BP, according to the Guide of European Society of Hypertension (ESH)/ European Society of Cardiology (ESC) [9].

High $\mathrm{BP}$ is an important cardiovascular risk factor [10]; an increase of $20 / 10 \mathrm{mmHg}$ of the systolic or diastolic pressure values causes doubling of the cardiovascular risk.

Additionally, the relationship between BP and the probability of developing ischemic heart disease and stroke is rising and progressive [11]. 


\section{References}

1. Kasper DL, Braunwald E, Fauci A, Hauser S, Longo D, Jameson JL. Harrison's Principles of Internal Medicine, 16th edition. New York: McGraw-Hill Medical Publishing Division, 2005.

2. Aeschbacher BC, Hutter D, Fuhrer J, et al. Diastolic dysfunction precedes myocardial hypertrophy in the development of hypertension. Am J Hypertens 2001; 14(2):106-13.

3. Staessen JA, Wang JG, Thijs L. Cardiovascular protection and blood pressure reduction: a meta-analysis. Lancet 2001; 358:1305-1315.

4. Lewis EF, Moye LA, Rouleau JL, Sacks FM, et al. Predictors of late development of heart failure in stable survivors of myocardial infarction: the CARE study. J Am Coll Cardiol 2003; 42:446-1453.

5. Richards AM, Nicholls MG, Troughton RW, Lainchbury JG, et al. Antecedent hypertension and heart failure after myocardial infarction. $J$ Am Coll Cardiol 2002; 39:1182-1188.

6. Nissen SE, Tuzcu EM, Schoenhagen $P$, et al. Effect of intensive compared with moderate lipid lowering therapy on progression of coronary atherosclerosis: a randomized controlled trial. JAMA 2004; 291:1071-80.

7. Nissen SE, Nicholls SJ, Sipahi I, et al. Effect of very high intensity statin therapy on regression of coronary atherosclerosis. The ASTEROID trial. JAMA 2006; 295:1556-65.

8. Task Force Members, Montalescot G, Sechtem $\mathrm{U}$, et al. ESC guidelines on the management of stable coronary artery disease. Eur Heart $J$ 2013; 34:2949-3003.

9. Task Force Members, Mancia G, Fagard R, et al. $2013 \mathrm{ESH} / \mathrm{ESC}$ Guidelines for the management of arterial hypertension: The Task Force for the management of arterial hypertension of the European Society of Hypertension (ESH) and of the European Society of Cardiology (ESC). Eur Heart J 2013; 34:2159-2219.

10. Gueyffier F, Bulpit C, Boissel JP, et al. Antihypertensive drugs in very old people: a subgroup meta-analysis of randomized controlled trials. Lancet 1999; 353(9155):793-6.

11. Lewington S, Clarke R, Qizilbash N, Peto R, Collins R; Prospective Studies Collaboration. Age-specific relevance of usual blood pressure to vascular mortality: a meta-analysis of individual data for one million adults in 61 prospective studies. Lancet 2002; 360(9349):1903-13. 